\title{
Entering and Exiting the Protein-Polyelectrolyte Coacervate Phase via Nonmonotonic Salt Dependence of Critical Conditions
}

\author{
Margarita Antonov, Malek Mazzawi, and Paul L. Dubin* \\ Department of Chemistry, 710 North Pleasant Street, University of Massachusetts, \\ Amherst, Massachusetts 01003
}

Received August 5, 2009; Revised Manuscript Received October 16, 2009

\begin{abstract}
Critical conditions for coacervation of poly(dimethyldiallylammonium chloride) (PDADMAC) with bovine serum albumin were determined as a function of ionic strength, $\mathrm{pH}$, and protein/polyelectrolyte stoichiometry. The resultant phase boundaries, clearly defined with this narrow molecular weight distribution PDADMAC sample, showed nonmonotonic ionic strength dependence, with the $\mathrm{pH}$-induced onset of coacervation $\left(\right.$ at $\left.\mathrm{pH}_{\phi}\right)$ occurring most readily at $20 \mathrm{mM} \mathrm{NaCl}$. The corresponding onset of soluble complex formation, $\mathrm{pH}_{\mathrm{c}}$, determined using highprecision turbidimetry sensitive to changes of less than $0.1 \%$ transmittance units, mirrored the ionic strength dependence of $\mathrm{pH}_{\phi}$. This nonmonotonic binding behavior is attributable to simultaneous screening of short-range attraction and long-range repulsion. The similarity of $\mathrm{pH}_{\mathrm{c}}$ and $\mathrm{pH}_{\phi}$ was explained by the effect of salt on protein binding, and consequently on the number of bound proteins relative to that required for charge neutralization of the complex, a requirement for phase separation. Expansion of the coacervation regime with chitosan, a polycation with charge spacing similar to that of PDADMAC, could be due to either the charge mobility or chain stiffness of the former. The $\mathrm{pH}_{\phi}$ versus $I$ phase boundary for PDADMAC correctly predicted entrance into and egress from the coacervation region by addition of either salt or water. The ability to induce or suppress coacervation via protein/polyelectrolyte stoichiometry $r$ was found to be consistent with the proposed model. The results indicate that the conjoint effects of $I, r$, and $\mathrm{pH}$ on coacervation could be represented by a three-dimensional phase boundary.
\end{abstract}

\section{Introduction}

The mixing of solutions of two hydrophilic colloids under suitable conditions can lead to liquid-liquid phase separation, also known as complex coacervation. ${ }^{1-3}$ The dilute (supernatant) phase is in equilibrium with the dense (coacervate) phase which is easily observed by microscopy or by centrifugation. This coacervate phase, formed by desolvation ${ }^{1}$ frequently occurs when electrostatic attractive forces overcome the hydration of the two particles. Such complex coacervation occurs with the neutralization of two oppositely charged macroions, in which at least one is a polyelectrolyte, while the other may also be a colloidal particle, such as a micelle or protein. In polyelectrolyteprotein systems, coacervation occurs when this charge neutralization is accomplished under conditions at which the $\mathrm{pH}$, relative to the protein isoelectric point, provides sufficient protein charge to bind to and neutralize the polyelectrolyte. This condition, sufficiently well delineated to be clearly identified by experiment, is referred to as $\mathrm{pH}_{\phi} \cdot{ }^{4,5} \mathrm{pH}_{\phi}$ corresponds to a very sharp transition, inasmuch as it may appear to be complete within a very small change in $\mathrm{pH}$, but it is susceptible to broadening by system polydispersity. For this reason we utilize a narrow-distribution polyelectrolyte and a well-characterized and pure protein so as to permit the clear identification of coacervation conditions. Coacervation is observed when the aggregation of soluble polymer-protein complexes is effectively infinite and is accompanied by desolvation with the appearance of an interface. This phase separation invariably arises from soluble complexes, which are typically multipolymer soluble aggregates, themselves developing from complexes of single

* To whom correspondence should be addressed. E-mail: dubin@chem. umass.edu. polymer chains associated with numerous proteins. Such intrapolymer complexes must approach electrical neutrality to further assemble. ${ }^{6,7}$ The formation of intrapolymer complexes, while not a true phase transition, appears as a discontinuity in such properties as scattering intensity, diffusivity, and electrophoretic mobility and is designated as $\mathrm{pH}_{\mathrm{c}}{ }^{5,6,8,9}$

While $\mathrm{pH}_{\phi}$ depends on polyelectrolyte molecular weight, $\mathrm{MW}$, the protein and polymer concentrations (stoichiometry), $\mathrm{pH}_{\mathrm{c}}$, are influenced solely by ionic strength. This is because $\mathrm{pH}_{\mathrm{c}}$, corresponding to the formation of soluble complexes, is governed only by the interactions between a charged domain on the protein and its associating sequence of polyelectrolyte charges. This is a local interaction, not influenced by events elsewhere along the polymer chain or events on other chains, and is therefore not affected by chain length or macromolecular concentrations. On the other hand, $\mathrm{pH}_{\phi}$ involves also long-range interactions between one soluble complex and another. These interactions are strongly controlled by the net charge on the soluble complex, $Z_{\mathrm{T}}$, which if too large can by repulsion prevent such interactions. The net charge is approximately (neglecting counterions) $Z_{\mathrm{T}}=Z_{\mathrm{P}}+n Z_{\mathrm{pr}}$, where $Z_{\mathrm{P}}$ is the total polyelectrolyte charge and $n$ is the number of proteins of charge $Z_{\mathrm{pr}}$, bound per polyelectrolyte chain. The condition for coacervation, $Z_{\mathrm{T}} \sim 0$ requires that $Z_{\mathrm{P}}$ and $Z_{\mathrm{pr}}$ are of opposite sign and $n$ is sufficiently large; however, initial binding may occur when $Z_{\mathrm{pr}}$ is of the same sign because of "patch binding" 8 to be discussed below. While $\mathrm{pH}$ and ionic strength alone determine the local protein-polyelectrolyte electrostatic affinity, $n$ follows a binding isotherm and depends on protein/polyelectrolyte bulk stoichiometry. The ability of complexes to associate even when their net charge $Z_{\mathrm{T}}$ is not precisely zero increases with the molecular weight of the complex and hence with polyelectrolyte MW due 
to polarization and disproportionation ${ }^{10}$ to be discussed further below. Intermediate between $\mathrm{pH}_{\mathrm{c}}$ and $\mathrm{pH}_{\phi}$ we may find intrapolymer soluble complexes (soluble aggregates) for which $Z_{\mathrm{T}}$ was close enough to zero to allow for soluble interpolymer complexes, but further charge accumulation prevents additional aggregation to form coacervate.

Both $\mathrm{pH}_{\mathrm{c}}$ and $\mathrm{pH}_{\phi}$ exhibit strong ionic strength dependence. An increase in ionic strength requires a concomitant increase in protein-polyelectrolyte electrostatic interaction to overcome screening. Depending on polyelectrolyte charge sign, this can correspond to an increase or decrease in the $\mathrm{pH}$ required for binding $\left(\mathrm{pH}_{\mathrm{c}}\right)$ and therefore to an increase or decrease in $\mathrm{pH}_{\phi}$ as well. The dependences of $\mathrm{pH}_{\mathrm{c}}$ and $\mathrm{pH}_{\phi}$ on ionic strength $I$ can be viewed as "phase boundaries", separating the regions of coacervation, soluble complex formation, and noninteraction ${ }^{11}$ (see Figure 2). Based on opposing effects of screening by salt and polyelectrolyte-protein interaction, one would expect a monotonic dependence of $\mathrm{pH}_{\mathrm{c}}$ on ionic strength; however, minima or at least dicontinuities in $\mathrm{pH}_{\mathrm{c}}$ with respect to $\underline{I}$ have been observed. ${ }^{12}$ These discontinuities in $\mathrm{pH}_{\mathrm{c}}$ correspond to maxima in binding affinity with respect to $I$ in the range of $I=$ $10-40 \mathrm{mM}^{13}$ at these ionic strengths the Debye screening length $\kappa^{-1}$ is close to the protein radius. ${ }^{12}$ This phenomenon has been explained in terms of short-range electrostatic attraction between the polyelectrolyte and a local "charge patch" coupled with longrange repulsion between the polyelectrolyte and the globally like-charge protein.

While nonmonotonic salt effects on $\mathrm{pH}_{\mathrm{c}}$ have been reported, the ionic strength dependence of $\mathrm{pH}_{\phi}$ is less clear, especially at low $I$. Mattison reported a discontinuity in $\mathrm{pH}_{\phi}$ for BSA and PDADMAC around $40 \mathrm{mM} \mathrm{NaCl} .^{11}$ Donati et al. ${ }^{14}$ found destabilization of a chitosan-alginate coacervate at ionic strengths outside of the range $0.02-0.10 \mathrm{M}$. Most recently Sperber et al. ${ }^{15}$ observed a maximum in $\mathrm{pH}_{\phi}$ at $I=30 \mathrm{mM}$ for mixtures of BLG and high charge density pectin. In general, the universality of such nonmonotonic behavior and its possible correlation with similar effects on $\mathrm{pH}_{\mathrm{c}}$ is not clear. A better understanding of this relationship could improve the possibility of manipulating phase behavior in polyelectrolyte-protein systems used for protein purification, ${ }^{16-18}$ drug delivery, ${ }^{19-21}$ microencapsulation, ${ }^{22}$ enzyme immobilization, ${ }^{23,24}$ and the formation and stabilization of food emulsions. ${ }^{25,26}$

In this paper we deal with the coacervation of bovine serum albumin with poly(dimethyldiallylammonium chloride), a system which we have studied intensively with respect to both complexation ${ }^{9,11}$ and coacervation. ${ }^{27-30}$ The general observation of minimal protein structural perturbation during complexation and coacervation including minimal loss of enzyme activity ${ }^{31}$ is supported in the BSA-PDADMAC system by minimal perturbation of protein titration curves in complexes. ${ }^{9}$ In the case of this strong cationic polyelectrolyte, we confirm nonmonotonic ionic strength dependence for transitions both from noninteraction to soluble complex and from soluble complex to coacervate, corresponding to minima in both $\mathrm{pH}_{\mathrm{c}}$ and $\mathrm{pH}_{\phi}$. The latter effect corresponds to entry into or exit from the coacervation region with changing salt concentration $I$. This same minimum in $\mathrm{pH}_{\phi}$ is observed when PDADMAC is replaced by chitosan, a polycation with the same structural charge density as PDAMAC; but differences in behavior are observed and discussed in terms of the lower persistence length and $\mathrm{pH}$-dependence of PDADMAC. The parallel behavior of $\mathrm{pH}_{\mathrm{c}}$ and $\mathrm{pH}_{\phi}$ is accounted for in terms of the effect of ionic strength on protein binding affinity and consequent influence on protein-polyelectrolyte microstoichiometry and complex electroneutrality. The dependence of complex microstoichiometry on bulk stoichiometry introduces the protein/polyelectrolyte weight ratio $r$ as an additional variable. We also discuss the relationship of $r$ to $I$ and $\mathrm{pH}$ at critical coacervation conditions, and we propose work that could lead to a three-dimensional coacervation phase boundary.

\section{Experimental Section}

Materials. Poly(dimethyldiallyammonium chloride) (PDADMAC) of $M_{\mathrm{w}}=219 \mathrm{kDa}\left(M_{\mathrm{n}}=141 \mathrm{kDa}\right)$ and $M_{\mathrm{w}}=700 \mathrm{kDa}\left(M_{\mathrm{n}}=460\right.$ $\mathrm{kDa}$ ) samples were prepared by free radical aqueous polymerization of diallydimethylammonium chloride ${ }^{32}$ and characterized after dialysis and lyophilization by membrane osmometry and light scattering. Chitosan was prepared by homogeneous de- $N$-acetylation of shrimp chitin as previously described and converted to the $\mathrm{HCl}$ salt ${ }^{33,34}$ and then lyophilized. Characterization by osmometry, viscosity measurement, and ${ }^{1} \mathrm{H}$ NMR revealed the following properties, respectively: $M_{\mathrm{n}}$ $=150 \mathrm{kDa},[\eta]=600 \mathrm{~mL} / \mathrm{g}$, and degree of acetylation $<0.1 \%$. Because coacervation behavior appeared particularly sensitive to the last variable, it was considered important to minimize this source of heterogeneity. Bovine serum albumin (BSA; $M_{\mathrm{w}} \approx 68 \mathrm{kDa}$ ) with total free acid content $\leq 1.2 \mathrm{mg} / \mathrm{g}$ was purchased from Roche Diagnostics (Indianapolis, IN; CAS 9048-46-8). $\mathrm{NaCl}$, sodium acetate, and standard $\mathrm{NaOH}, \mathrm{HCl}$, and acetic acid solutions were from Fisher Scientific. Milli-Q water was used in all sample preparation.

Determination of $\mathbf{p H}_{\mathbf{c}}$ and $\mathbf{p} \mathbf{H}_{\phi}$. PDADMAC solutions $(0.12 \mathrm{~g} / \mathrm{L})$ and BSA solutions $(0.6 \mathrm{~g} / \mathrm{L})$ were prepared separately at the desired concentration of $\mathrm{NaCl}(5-400 \mathrm{mM})$ and filtered $(0.22 \mu \mathrm{m}$ AcetatePlus, Osmonics Inc.). The $\mathrm{pH}$ of each solution was adjusted to $3.5-4$ (noninteracting conditions) with $0.1 \mathrm{~N} \mathrm{HCl}$ so that initial mixing would be completely homogeneous. Turbidimetric titration was carried out by addition $0.01 \mathrm{M} \mathrm{NaOH}$ to a total solution volume of $10.0 \mathrm{~mL}$ in increments of $0.002 \mathrm{~mL}$ with stirring and simultaneous monitoring of $\mathrm{pH}$ and transmittance. \% Transmittance was measured using a Brinkmann PC 800 colorimeter equipped with a $2 \mathrm{~cm}$ path length fiber optics probe and a $450 \mathrm{~nm}$ filter, and $\mathrm{pH}$ was measured with a Corning 340 $\mathrm{pH}$ meter, both integrated into a system of our own design which is programmed for (1) automated delivery of selected titrant volumes via a $2 \mathrm{~mL}$ Gilmont microburet at selected rates of addition, (2) the number of transmittance and $\mathrm{pH}$ readings to be averaged, and (3) the terminal $\mathrm{pH}$. After conventional calibration of the $\mathrm{pH}$ meter, the colorimeter is calibrated with pure water, setting $\% T=100$, and with a latex suspension of known turbidity (ca. 30\%T). Coacervation at $\mathrm{pH}_{\varphi}$ is clearly evident from an abrupt visible increase in turbidity corresponding to an increase in $100-\% T=\tau^{\prime}$. The onset of complex formation at $\mathrm{pH}_{\mathrm{c}}$ is more subtle, and is determined by the intersection between two ranges. The first range at low $\mathrm{pH}$ is one in which (1) $\tau^{\prime}$ remains below $\tau^{\prime}=3$, and constant within \pm 0.1 transmittance (this corresponds to scattering due to the protein); and (2) the best fit line of all data points has a slope indistinguishable from zero within experimental error. The second region, corresponding to initial soluble complex formation, is defined by a best fit line of positive slope from which all data points deviate by less than $0.2 \% \mathrm{~T}$. The measurement of $\mathrm{pH}_{\mathrm{c}}$ via small changes in $\tau^{\prime}$, close to $100 \%$ transmittance, require high precision and sensitivity. The low drift, high sensitivity, and low noise (due to signal average) with this instrument make it possible to detect those subtle changes normally observed with dynamic light scattering.

Turbidimetric Titrations at Fixed $\mathbf{p H}$ and Varying Ionic Strength. To confirm values of $\mathrm{pH}_{\varphi}$ obtained at fixed ionic strength $I$, we determined the point of coacervation by varying $I$ at fixed $\mathrm{pH}$. Solutions were prepared as above but in pure water with final adjustment of $\mathrm{pH}$ to $7.1 \pm 0.15$. Turbidimetric titrations (nonautomated) were carried out in duplicate by adding $4 \mathrm{M} \mathrm{NaCl}$ with continuous stirring to the solutions of mixed PDADMAC and BSA and using a $4.0 \mathrm{~cm}$ path length fiber-optics probe with a $420 \mathrm{~nm}$ filter to measure $\tau^{\prime}$. Turbidity values were recorded when the meter response stabilized 

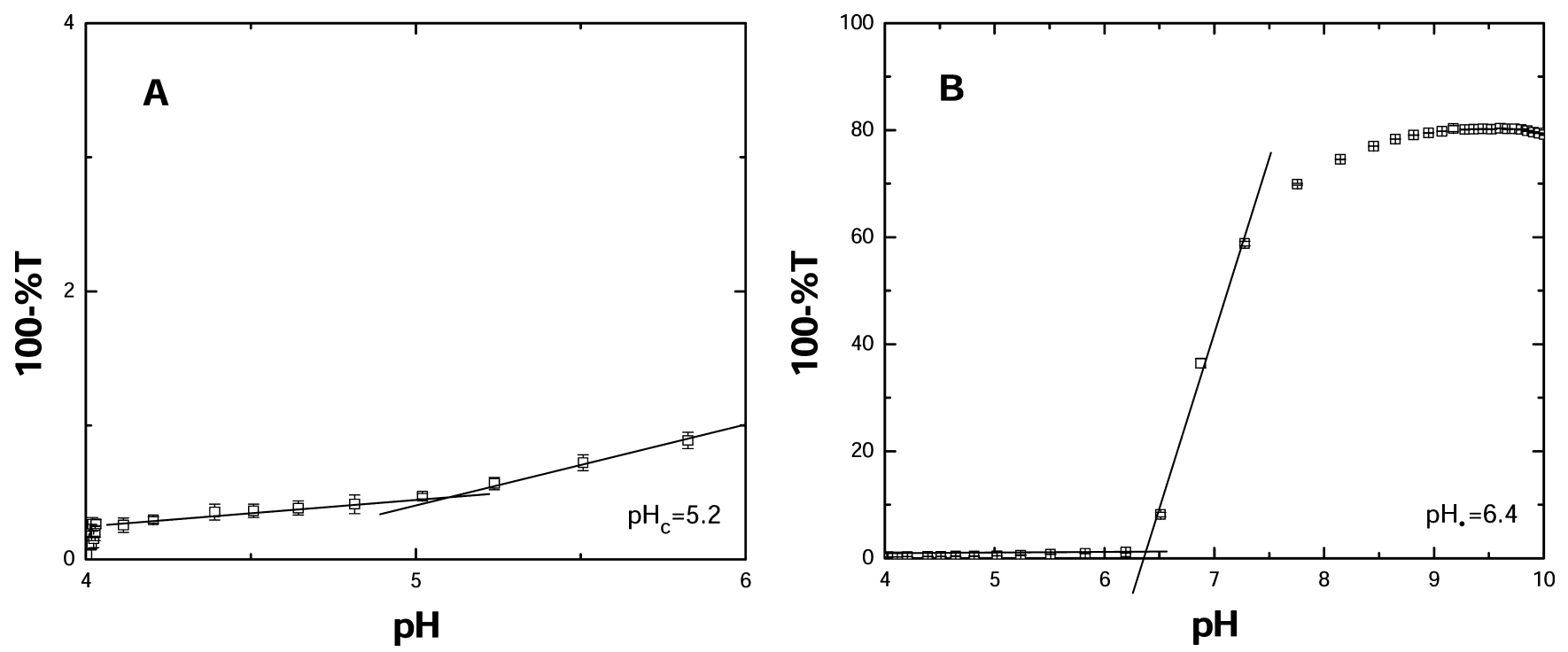

Figure 1. Turbidimetric determination of transitions for BSA-PDADMAC in $50 \mathrm{mM} \mathrm{NaCl}$ corresponding to complex formation (A) and coacervation (B). Lines show definitions of $\mathrm{pH}_{\mathrm{c}}$ and $\mathrm{pH}_{\phi} ;(\mathrm{A})$ is an expansion of the low $\mathrm{pH}$ region of $(\mathrm{B})$. Data points obtained automatically at $\mathrm{pH} 4$ represent stabilization of the probe prior to titration. Nonzero slope at $\mathrm{pH}<5$ may be attributable to shift in BSA monomer-dimer equilibrium.

within a value of $0.1 \% T$. To test reversibility a solution adjusted to be within the coacervate region (e.g., pH 7.15 and $20 \mathrm{mM}$ ) was titrated with pure water. We also demonstrated exit from the coacervate region from high $(20 \mathrm{mM}) \mathrm{NaCl}$ conditions, by addition of pure water at fixed $\mathrm{pH}$.

Stoichiometric Titrations. "Type 2" turbidimetric titrations were previously defined as the addition of charged colloidal particle to polyelectrolyte, ${ }^{35}$ here at fixed $\mathrm{pH}$ and $I$. A solution of $0.12 \mathrm{~g} / \mathrm{L}$ PDADMAC was prepared at $\mathrm{pH} 7.9 \pm 0.2$ and $I=10 \mathrm{mM}$ and manually titrated with $20 \mathrm{~g} / \mathrm{L} \mathrm{BSA}$ in $10 \mathrm{mM} \mathrm{NaCl}$, adjusted to $\mathrm{pH}$ 7.86 .

\section{Results and Discussion}

1. Coacervation Phase Boundary. Values of the critical $\mathrm{pH}$ for soluble complex formation $\left(\mathrm{pH}_{\mathrm{c}}\right)$ and coacervation $\left(\mathrm{pH}_{\varphi}\right)$ were determined by the addition of $\mathrm{NaOH}$ to an acidic solution of BSA and PDADMAC, typically with weight ratio $r=5$, with results shown in Figure 1. Two transitions may be identified, as shown in Figure 1A,B, with the first corresponding to the onset of the formation of soluble complexes and the second corresponding to incipient phase separation, designated here and elsewhere ${ }^{36,37}$ as $\mathrm{pH}_{\mathrm{c}}$ and $\mathrm{pH}_{\phi}$, respectively. The very low and constant values for turbidity seen at $\mathrm{pH}<\mathrm{pH}_{\mathrm{c}}$ demonstrate the absence of BSA aggregation at conditions where it might be expected, that is, low ionic strength and close to $\mathrm{pI}$, where such "isoelectric precipitation" was seen in "blank titrations" of BSA alone. From such turbidimetric titrations we obtained the phase boundaries in Figure 2, as $\mathrm{pH}_{\varphi}$ and $\mathrm{pH}_{\mathrm{c}}$ versus $I^{1 / 2}$. We use this abscissa because $I^{1 / 2}$ is nearly equal to $0.3 \kappa$, where $\kappa^{-1}$ is the Debye length in $\mathrm{nm}$ and because it more clearly delineates important features at low $I$, particularly the well-defined minimum in $\mathrm{pH}_{\varphi}$ at $I^{1 / 2}=0.13(17 \mathrm{mM})$. This striking feature, duplicated by the minimum in $\mathrm{pH}_{\mathrm{c}}$, is the central theme of this paper. Similar nonmonotonic behavior of $\mathrm{pH}_{\varphi}$ observed, but not discussed, by Sperber et al. ${ }^{15}$ for pectin (a polyanion) with $\beta$-lactoglobulin, suggests that this behavior may be general for polyelectrolyte-protein systems. Other notable features of Figure 2 are the absence of coacervation at any ionic strength for $\mathrm{pH}<5.8$; the appearance of a parallel minimum in $\mathrm{pH}_{\mathrm{c}}$; and the increase in the range of ionic strength over which coacervates are stable with increase in $\mathrm{pH}$. We proceed to address these salient features below.

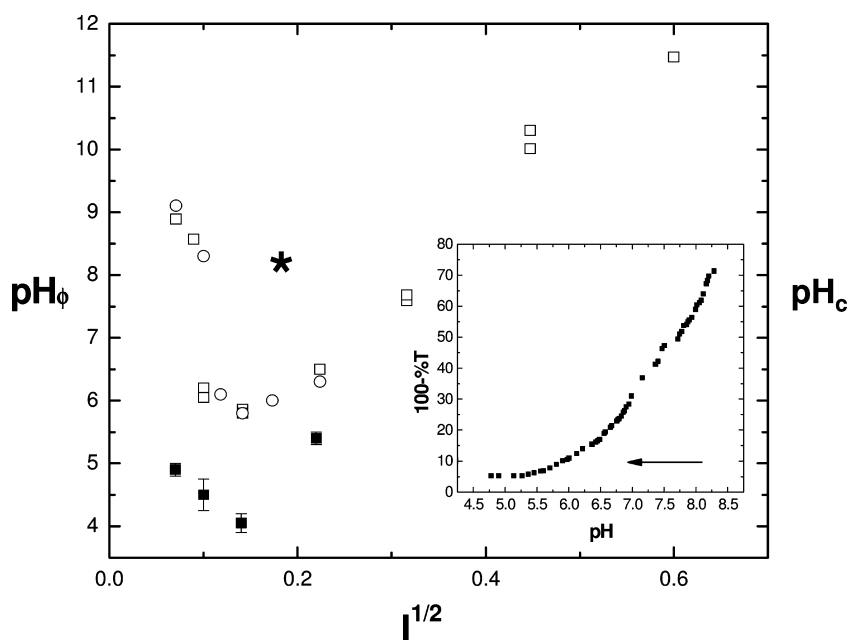

Figure 2. Phase boundaries for BSA-PDADMAC: $\mathrm{pH}_{\phi}(\theta, \mu)$, different protein lots, axis left; $\mathrm{pH}_{\mathrm{c}}(v)$, axis right. Inset: turbidity change as the phase boundary is exited by addition of $\mathrm{HCl}$ at fixed $I^{1 / 2}=0.17$ (30 $\mathrm{mM}$ ). Protein and polymer concentrations: 0.60 and $0.12 \mathrm{~g} / \mathrm{L}$. Asterisk in main plot represents starting $\mathrm{pH}$ and ionic strength for the inset titration.

The $\mathrm{pH}$-induced transitions into the coacervation region (see Figure 1) used to generate the $\mathrm{pH}_{\varphi}$ phase boundary of Figure 2 appear to be quite abrupt. To test reversibility, we brought the system to coacervation at $\mathrm{pH} 8.3$ at $I^{1 / 2}=0.17(30 \mathrm{mM}$; corresponding to the asterisk in Figure 2) and then titrated with $\mathrm{HCl}$ to $\mathrm{pH} 4.8$. The consequent reemergence from the coacervate region with decreasing $\mathrm{pH}$ shown in the inset of Figure 2 reveals limited reversibility: low turbidity is attained around $\mathrm{pH} 5.5$, as expected from Figure 2. The limiting value of $100-\% T<$ about 5 is consistent with soluble complex, but the transition is gradual relative to that seen in Figure 1B, which might signify different kinetics for coacervate dissolution as compared to coacervate formation.

A notable feature in Figure 2 is the corresponding minima in $\mathrm{pH}_{\mathrm{c}}$ and $\mathrm{pH}_{\phi}$. A discontinuity in $\mathrm{pH}_{\mathrm{c}}$ at a value of $I$ corresponding to a Debye length $\kappa^{-1}$ close to the protein radius has been previously noted; this was interpreted as a discontinuity in the ionic strength dependence of the binding affinity and found to be a general feature for proteins binding to polyelec- 


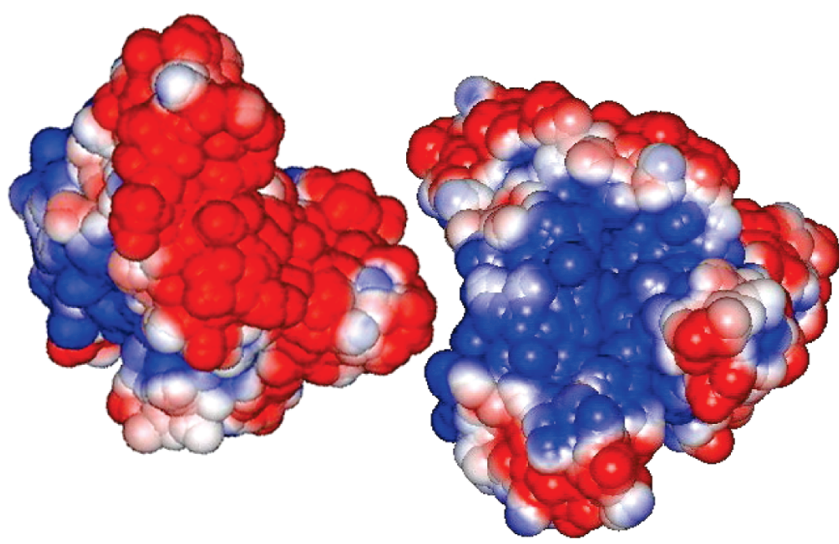

Figure 3. Charge anisotropy of BSA at $\mathrm{pH} 7$, represented by Delphi images (two views) showing regions with electrostatic potential of $+0.1 \mathrm{kT}$ (blue) and $-0.1 \mathrm{kT}$ (red; see ref 12 for details of Delphi calculation and visualization).

trolytes of like charge by Seyrek et al., who observed for a number of protein-polyelectrolyte systems binding constants attaining maximum values in the ionic strength $10-40 \mathrm{mM}$, which includes the minima seen in Figure 2. ${ }^{12}$ Such nonmonotonic effects were explained as arising from a combination of short-range attraction and long-range repulsion when polyelectrolytes bind to proteins with charge anisotropy; ${ }^{12}$ such charge anisotropy is demonstrated for BSA near $\mathrm{pH} 7$ by the Delphi images in Figure 3. The prominent positive domain appears at $\mathrm{pH} \mathrm{7,} \mathrm{as} \mathrm{described} \mathrm{in} \mathrm{ref} 12$; the same computational result is presented in a more visual manner in Figure 3 . To correlate the minima in $\mathrm{pH}_{\mathrm{c}}$ and $\mathrm{pH}_{\phi}$, it is necessary to relate binding affinity to coacervation, and we do so by considering coacervation as a consequence of charge neutrality of the protein-polyelectrolyte complex. Thus, neglecting counterions, the net complex charge $Z_{\mathrm{P}}+n_{\mathrm{pr}} Z_{\mathrm{pr}}=Z_{\mathrm{T}} \approx 0$ at coacervation, so that $Z_{\mathrm{P}} / Z_{\mathrm{pr}}=n^{*}{ }_{\mathrm{pr}}$, where $n^{*}$ pr is the number of proteins bound at $\mathrm{pH}=\mathrm{pH}_{\phi}$. The fact that coacervate is stable over a range of $I$ in most of the coacervation domain (see Figure 2) means that some range of $n_{\mathrm{pr}}{ }^{*}$ provides sufficient proximity to the condition for coacervation. The formation of a condensed phase at an average complex charge that deviates from zero has been accounted for by Shklovskii and Zhang in terms of inter- or intracomplex disproportionation. ${ }^{10}$ The influence of ionic strength on binding at fixed $\mathrm{pH}$ is shown diagrammatically by the hypothetical binding isotherms in Scheme 1, here represented as the dependence of $n_{\mathrm{pr}}$ on added protein normalized as the weight ratio of protein to polymer, $r$. The shaded region designated by $n_{\mathrm{pr}}{ }^{*}$ corresponds to the range of net complex charges $Z_{\mathrm{T}}$ close enough to zero to permit coacervation. ${ }^{10}$ These curves, constructed to be qualitatively consistent with Figure 2, have two features: the total amount of protein bound at saturation (plateau) and the binding affinity (binding constant $K_{\mathrm{b}}$ ) proportional to the initial slopes of the isotherms. The curve in Scheme 1, designated as $20 \mathrm{mM}$, represents the expectation from Figure 2 of a maximum in $K_{\mathrm{b}}$ at this ionic strength, corresponding to a maximum in initial slope $\left(\mathrm{d} n_{\mathrm{pr}} / \mathrm{d} r\right)_{0}$. The nonmonotonic behavior results from the intermediate position of the lowest ionic strength ( $5 \mathrm{mM}$ ), and the placement of the $I=20 \mathrm{mM}$ curve in the position of steepest slope to be consistent with the characteristic ionic strengths of maximum binding affinity reported in ref 12 . The curves cross because effects of $I$ on the binding constant and on the saturation value of $n_{\text {pr }}^{\max }$ are not parallel, based on the assumption that $n_{\text {pr }}^{\max }$ decreases at low $I$ due to interprotein repulsion, so that the largest plateau value is seen for the highest ionic strength, $60 \mathrm{mM}$. A somewhat similar argument was put
Scheme 1. Hypothetical BSA-PDADMAC Binding Isotherms Represented as the Number of Proteins Bound per Polymer Chain $n_{\mathrm{pr}}$ vs Protein/Polymer Weight Ratio $r$, at an Arbitrary Fixed pH, and at Ionic Strengths Shown ${ }^{a}$

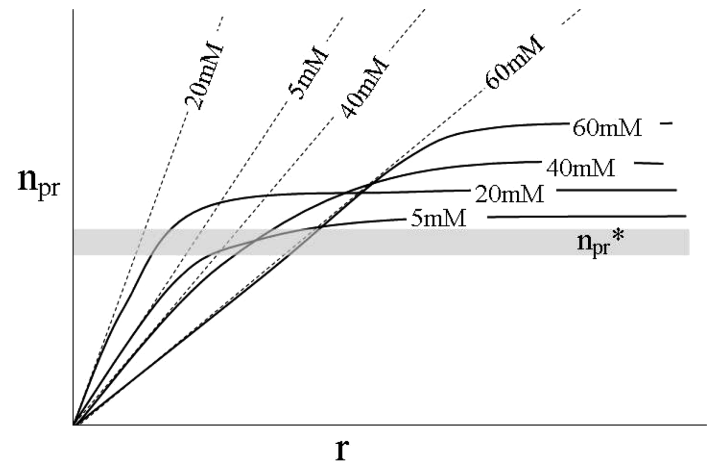

a These isotherms are drawn to be consistent with maximum binding at $I=20 \mathrm{mM}$ (Figure 2). Complex charge neutrality is attained when the number of bound proteins per chain $n_{\mathrm{pr}}=n^{\star}{ }_{\mathrm{pr}}$, at which point complex net charge is zero. Coacervation (shaded region) occurs over a range of $n_{\mathrm{pr}}$ (see text).

forward by Sperber et al. ${ }^{15}$ for the enhancement of coacervation by the addition of salt at low $I$, namely, an increase in $n_{\mathrm{pr}}$ due to the suppression of interprotein repulsion. The crossover point of the $5 \mathrm{mM}$ and $40 \mathrm{mM}$ curves within the shaded region means that identical values of $n_{\mathrm{pr}}=n_{\mathrm{pr}} *$ can exist at fixed $r$ for those two ionic strengths. Thus, at fixed $\mathrm{pH}$ and $r$, both values of $I$ can lie on the coacervation phase boundary, as we observe in Figure 2. At fixed $r$, either decreasing or increasing $I$ from a starting point of $20 \mathrm{mM}$ results in a decrease in $n_{\mathrm{pr}}$, so that complex charge becomes more positive and coacervate dissolves. The preceding argument may account for the correlation of minima in $\mathrm{pH}_{\mathrm{c}}$ and $\mathrm{pH}_{\varphi}$, but does not however establish that the minimum in $\mathrm{pH}_{\mathrm{c}}$ is a necessary or sufficient condition for the nonmonotonic behavior of $\mathrm{pH}_{\varphi}$.

A more direct "colloid-based" explanation of the nonmonotonic behavior could be offered if we view the species at incipient coacervation as a soluble complex aggregate with a nonzero net charge. Its ongoing association with other similar particles, driven by the elimination of exterior regions in favor of lower-energy, more efficiently ion-paired interior regions, would then be an example of a short-range attraction coupled with a long-range repulsion ("coulomb blocking"). Such behavior, in which attractive and repulsive interactions have different length scales, has been the subject of considerable recent interest. ${ }^{39-41}$ It is important to point out that among the short-range attraction/long-range repulsion (SALR) scenarios usually presented for colloidal systems, the "LR" interactions are invariably Coulombic, while the "SA" interactions usually arise from "depletion flocculation" 38 or some unidentified forces. ${ }^{40}$ In the present case, both interactions are Coulombic, attraction being short-range only because the preferred orientation of the protein relative to the bound polyelectrolyte makes it so. ${ }^{12}$

In these analyses, we consider the role of added $\mathrm{NaCl}$ to be purely one of Debye-Hückel screening, but specific $\mathrm{Cl}^{-}$ion binding to BSA has been established in several studies. ${ }^{42,43}$ The magnitude of this effect is small but not negligible; at $\mathrm{pH} 7$, for example, an increase in $[\mathrm{NaCl}]$ from 10 to $100 \mathrm{mM}$ (moving across the coacervate phase boundary) appears to reduce the charge on BSA from -12 to -14 . In principle, this might make breakup of complexes and coacervate more difficult, but this does not appear to be a significant factor.

Sperber et al. ${ }^{15}$ also observed nonmonotonic behavior of $\mathrm{pH}_{\varphi}$ for the coacervation of $\beta$-lactoblobulin with high charge-density 


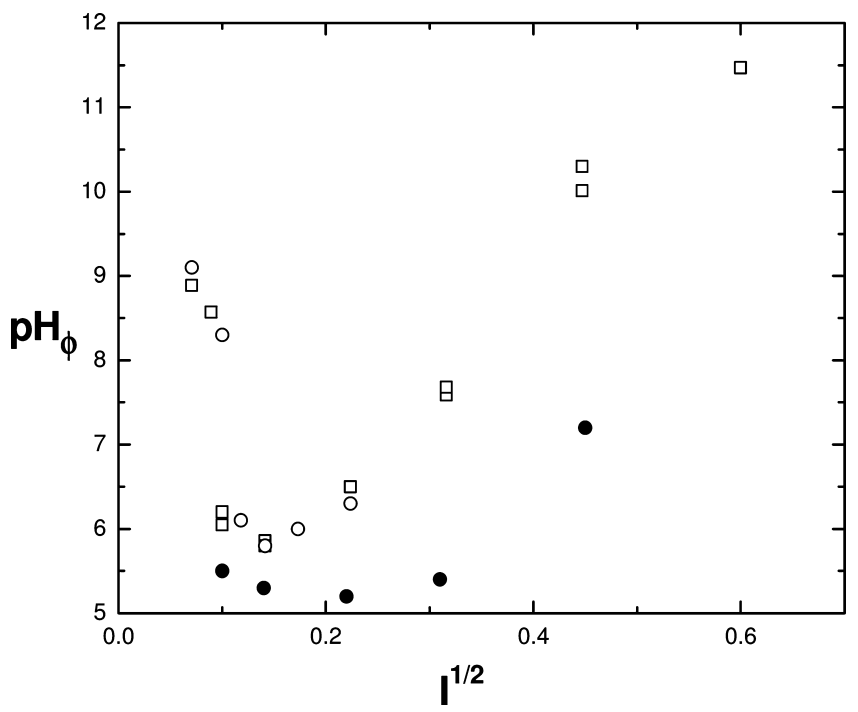

Figure 4. Phase boundaries for coacervation $(\mathrm{pH} \phi)$ for BSAPDADMAC (open symbols, $\theta, \mu$ from Figure 2 ) and BSA-chitosan $(\lambda)$. Conditions as for Figure 2.

(low methoxy) pectin (in the case of this anionic polyelectrolyte, a maximum in $\mathrm{pH}_{\varphi}$ at $I \cong 25 \mathrm{mM}$ ). In contrast to the present study, Sperber et al. did not observe a corresponding feature in $\mathrm{pH}_{\mathrm{c}}$, although a marked discontinuity in $\mathrm{pH}_{\mathrm{c}}$ appeared at this ionic strength. While the maximum in $\mathrm{pH}_{\varphi}$ was not addressed in ref 15 , it was suggested that the addition of salt could increase $n$ by reducing interprotein repulsions at $\mathrm{pH}<\mathrm{pI}$, similar to the behavior at large $r$ suggested in Scheme 1. It should be noted that results for pectin at $\mathrm{pH}<5.0$ could reflect its charge mobility, a significant factor at $\left(\mathrm{pH}-\mathrm{p} K_{\mathrm{a}}\right)<1 .{ }^{44}$

2. PDADMAC versus Chitosan. Chitosan-protein complexes have attracted considerable interest in a number of fields due to the biocompatibility of chitosan. ${ }^{45}$ Chitosan provides an interesting comparison to PDADMAC in that the average chemical spacing between ionophores is about $6 \AA$ for both, while the persistence length of chitosan of $6 \mathrm{~nm}$ is about 2-3 times larger than that of PDADMAC. Also, in contrast to PDADMAC, the charge of chitosan $Z_{\mathrm{P}}$ is $\mathrm{pH}$ dependent. We previously noted that chitosan forms coacervates with BSA in $100 \mathrm{mM} \mathrm{NaCl}$ (Debye length ca. $1 \mathrm{~nm}$ ) at a lower $\mathrm{pH}$ than PDADMAC $;^{29}$ that is, in a mixture of chitosan and PDADMAC, the former would coacervate first as the $\mathrm{pH}$ is increased from, for example, 4. Because this is opposite to the expectation of weaker binding due to greater chain rigidity, we have to explain the difference between PDADMAC and chitosan in terms of the $\mathrm{pH}$-dependence of chitosan charge. Figure 4 shows this expansion of the coacervate region (coacervation with chitosan at $\mathrm{pH}>7.5$ cannot be measured due to its loss of solubility). Chitosan coacervation also exhibits nonmonotonic salt effects with a minimum at $50 \mathrm{mM} \mathrm{NaCl}$, higher than the minimum for PDADMAC. Finally, the remarkably strong ionic strength dependence at low $I$ seen with PDADMAC is not seen with chitosan, which exhibits a shallow minimum.

The lower values of $\mathrm{pH}_{\phi}$ for chitosan may be explained by (1) a decrease in the number and charge of proteins that must be bound to achieve $Z_{\mathrm{T}}=0$ due to a decrease in $Z_{\mathrm{P}}$, (2) an increase in binding affinity so that $n_{\mathrm{pr}}$ is bigger for chitosan than for PDADMAC at fixed $\mathrm{pH}$, or (3) differences in properties of nearly neutral soluble complexes for the two polymers. With regard to $Z_{\mathrm{P}}$, the degree of protonation of chitosan begins to decrease at $\mathrm{pH}>5$, with a two-fold drop in the degree of protonation at $\mathrm{pH} 6.5 .^{46}$ With regard to protein-binding affinity, this same deprotonation goes hand-in-hand with charge mobility, allowing for the formation of positive charge patterns on chitosan complementary to the negative protein domains. Finally, coacervation with chitosan might occur at a complex net charge further from zero through a reduction in the loss of entropy when the dense phase is formed with more rigid complexes. Simulations by $\mathrm{Ou}$ and Muthukumar suggested that chitosan, as weak PE, might display a smaller role for counterion release entropy and larger role of favorable enthalpy. ${ }^{47}$ However, these effects do not influence the consideration of chain configurational entropy presented above. The competition between loss of chain configurational entropy and gain of counterion entropy has been discussed ${ }^{48}$ for a more closely related serum albumin-polycation system. Resolution among these manifold effects is not possible at present.

To explain the larger value of $I_{\min }$ with chitosan, we consider that the bound polycation at these low $\mathrm{pH}$ values experiences both short-range attraction to a negative protein domain and long-range repulsion from positive domains. ${ }^{12}$ As alluded to above, the condition of $I_{\text {opt }}$ for complex formation occurs when the balance between screening of short-range attractions and long-range repulsions is optimal, and this occurs when the Debye length falls between the two relevant lengths, for attraction and repulsion, respectively. This optimal Debye length will be larger when the characteristic $\mathrm{PE}$ - protein distance for repulsions is larger, as is the case for PDADMAC since chain flexibility allows those repelled segments to distance themselves from the protein. This larger Debye length corresponds to a lower optimal ionic strength for PDADMAC.

The effect of ionic strength on $\mathrm{pH}_{\phi}$ is smaller for chitosan than for PDADMAC. This is especially visible at values of low $I$, where the increase in $\mathrm{pH}_{\phi}$ with decreasing $I$ is very large for PDADMAC. To explain this, we relate the enhanced coacervation for chitosan to its ability to bind BSA more strongly due to charge mobility, which can maintain a high degree of chitosan protonation in proximity to the bound protein's negative domain, while reducing chitosan charges at more distal locations subject to repulsions from the protein's positive domains. This effect was previously demonstrated for other weak polyelectrolytes, namely, by comparison of carboxylated versus sulfonated polymers of equal linear charge density. ${ }^{44}$ In the absence of such repulsions, their screening by salt, which accounts for the negative slope at low $I$ in Figure 2, becomes less significant. In the region of high salt, where repulsions are fully suppressed, salt only screens attractions which differ little for protein-bound sequences of chitosan and PDADMAC. Therefore, the ionic strength effects in this region are quite similar, as evident from the slopes for the two polymers at $I>0.04 M$.

Turbidimetric titrations with chitosan, shown in Figure 5, differ markedly from those for PDADMAC, such as shown in Figure 1, most prominently in the appearance of a second maximum after $\mathrm{pH}_{\phi}$. Deprotonation of chitosan at $\mathrm{pH}>6^{49}$ leads to charge reversal of complexes as protein and polyelectrolyte become progressively more negative and less positive, respectively, leading to net-negative complexes and coacervate destabilization and dissolution. The peak or shoulder at $\mathrm{pH} 7-8$ corresponds to the phase separation of chitosan, which is $95 \%$ deprotonated at $\mathrm{pH}>7.5$. Athough the $\mathrm{MW}$ distribution of the chitosan sample has not been determined, the tendency of chitosan to aggregate is known to be highly sensitive to high MW species ${ }^{50}$ but polyelectrolyte-protein binding affinity is little affected by MW. ${ }^{11}$ The magnitude of coacervate turbidity decreases monotonically with increasing salt and disappears at $200 \mathrm{mM}$. It is likely that charge neutralization may be reached 


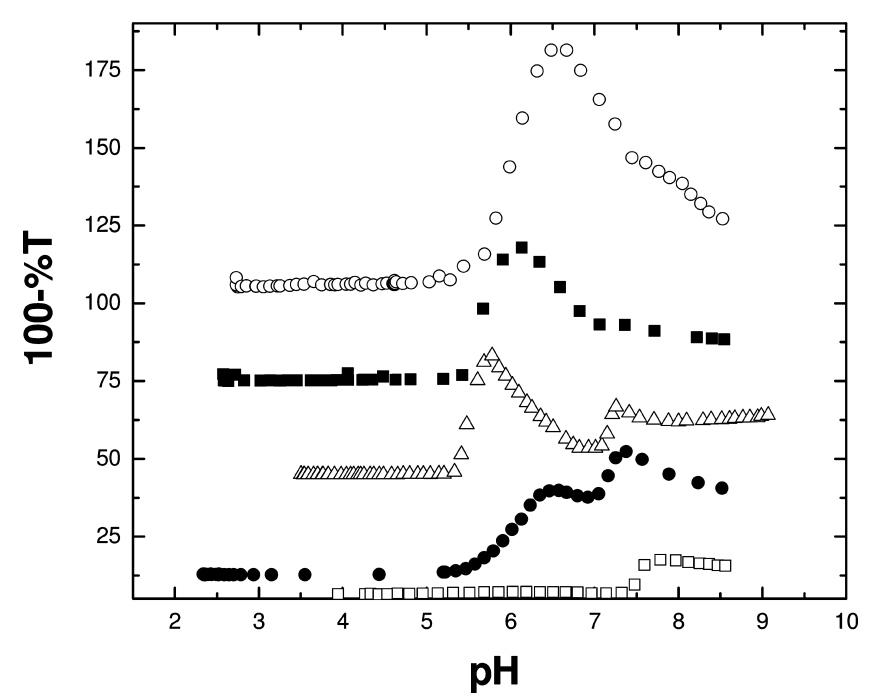

Figure 5. Turbidimetric $\mathrm{pH}$ titrations of $\mathrm{BSA} /$ chitosan (concentrations as for Figure 2) at ionic strength (from top to bottom): 10, 20, 50, 100 , and $200 \mathrm{mM}$. Arbitrary vertical shifts for clarity.

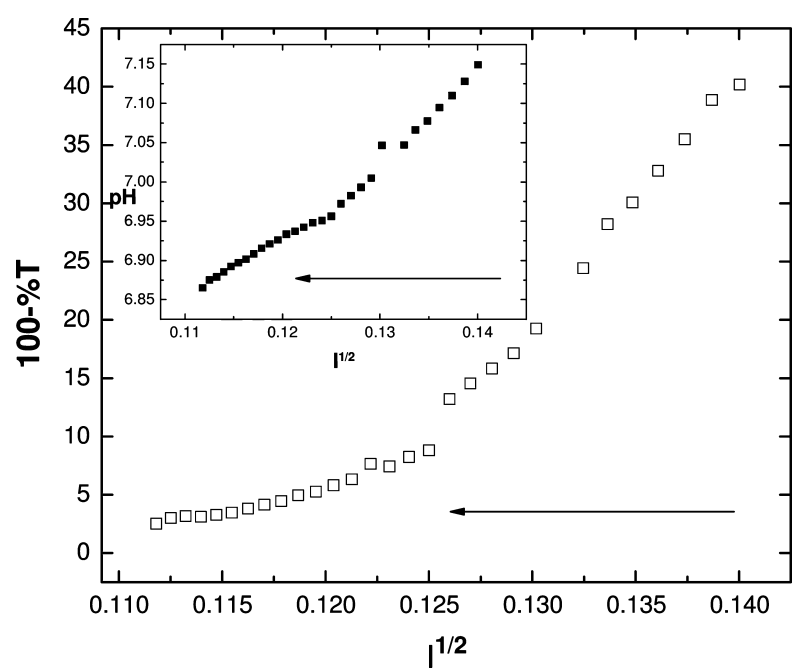

Figure 6. Effect of addition of water to BSA-PDADMAC coacervate at $\mathrm{pH} 7.15$ (concentrations as for Figure 2) as turbidity vs $I_{1 / 2}$. Inset: variation of $\mathrm{pH}$ with addition of water.

at some $\mathrm{pH}$ for all ionic strengths, but the level and strength of protein-polyelectrolyte interactions may be too weak to lead to counterion release, a likely driving force for coacervation. Alternatively, the concentrations of unbound proteins and chitosan may be large, thus reducing the quantity of coacervate. Additional measurements such as compositional analyses of supernatant and coacervate are planned to test these hypotheses.

3. Effect of Ionic Strength. To confirm the phase transitions of Figure 2, dilution with water at fixed $\mathrm{pH}$ was carried out from a starting point inside the coacervation region at $\mathrm{pH} 7.15$, $I^{1 / 2}=0.14(20 \mathrm{mM}$; see asterisk in Figure 2) with the result in Figure 6. As expected from Figure 2, emergence from the coacervate region is observed in the vicinity of $I^{1 / 2}=0.12(15$ $\mathrm{mM}$ ), but the approach to this state with decreasing $I$ appears to be gradual. The small change in $\mathrm{pH}$ with $I$ (inset) cannot account for this since its direction is toward diminished coacervation (lower $\mathrm{pH}$ ). Because the dissolution of coacervate could involve slow kinetics, a parallel experiment was carried out by addition of salt as shown in Figure 7A. Entry into coacervate occurs at $I^{1 / 2}=0.11(12 \mathrm{mM})$, at a somewhat lower ionic strength than exit from it in Figure 6, apparently more abruptly, suggesting the possibility of different kinetics for

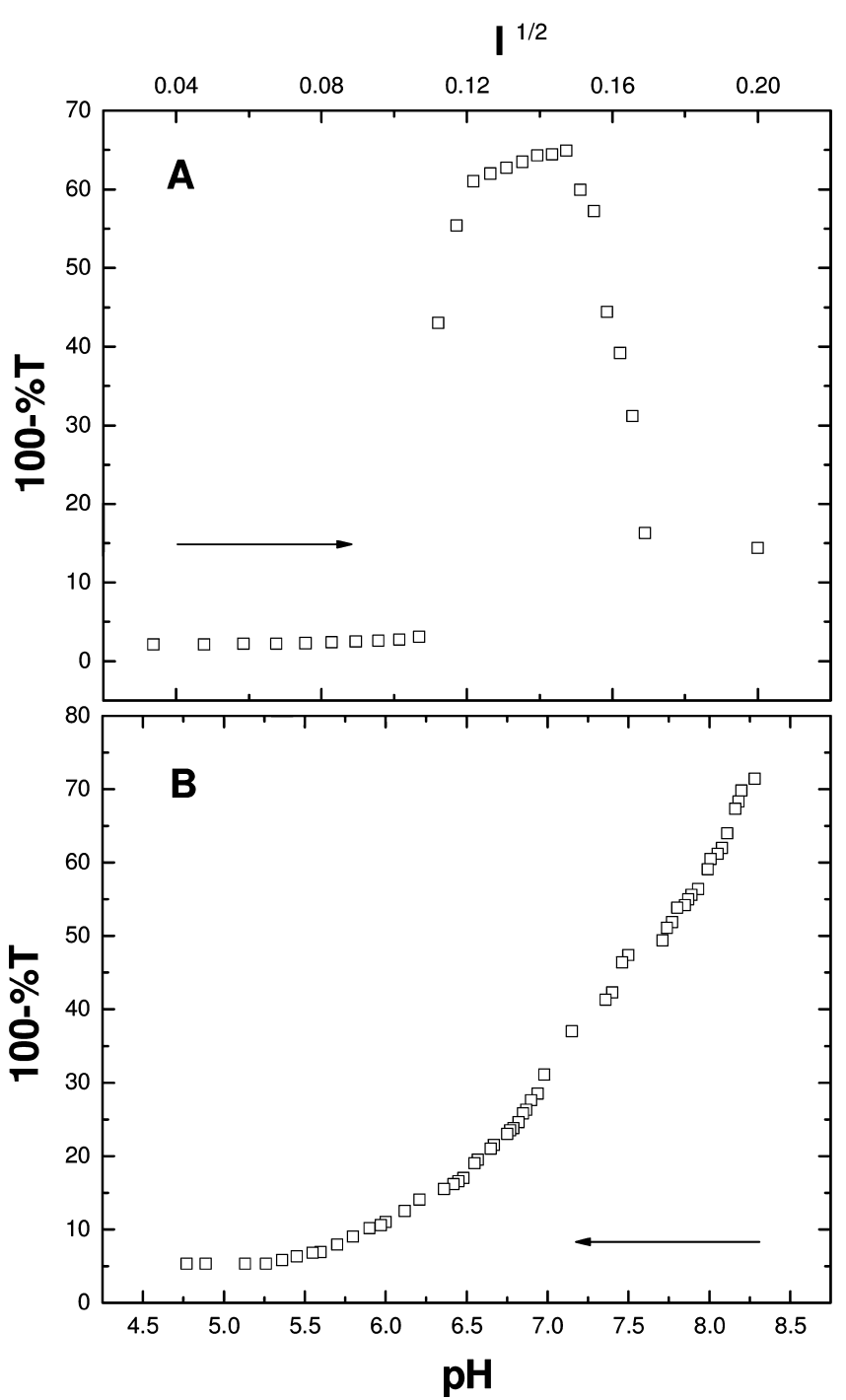

Figure 7. (A) Addition of $\mathrm{NaCl}$ to BSA-DADMAC (concentrations as for Figure 2) at $\mathrm{pH}=7.15$. (B) Gradual transition from the coacervate region to the soluble complex region by addition of $\mathrm{HCl}$ (left to right) at $I_{1 / 2}=0.17(30 \mathrm{mM})$.

coacervation vs dissolution; but in very good agreement with the corresponding position in Figure 2. Similar salt-induced entry and exit into coacervation has been reported for the interaction of alginate and lactose-modfied chitosan. ${ }^{14}$ Emergence from the coacervate region is seen in the range of $I^{1 / 2}=0.15-0.16$ $(22-27 \mathrm{mM})$ in contrast to the value of $I^{1 / 2}=0.26(70 \mathrm{mM})$ indicated by Figure 2. It is possible that the data points at high salt do not represent equilibrium values, as also indicated by the higher values of turbidity in the region of coacervate dissolution observed when using shorter time intervals between the added increments of $\mathrm{NaCl}$. Because coacervate dissolution depends on equilibration of $\mathrm{NaCl}$ between droplets and dilute phase, this portion of the titration curve in Figure 7A could depend on the size homogeneity of droplets, which was not determined. However, the more problematic aspect of comparing the high ionic strength region of Figure 7A (leaving the coacervate region) with the upward arm of the phase boundary of Figure 2 is that the latter corresponds to entering the coacervate region.

To what extent might the minimum in the coacervation boundary $\mathrm{pH}_{\phi}(I)$ of Figure 2 be a consequence of the minimum in the binding boundary, $\mathrm{pH}_{\mathrm{c}}(I)$ ? To answer this we begin by attempting to bring the two types of experiments (effect on 
coacervation of $\mathrm{pH}$ vs effect of $I$ ) into conformity by having both correspond to coacervate dissolution. This will enable us to evaluate the relative effects of $\mathrm{pH}$ and ionic strength on this transition and to then compare that to the relative effects of $\mathrm{pH}$ and ionic strength for the onset of binding. We first identify an increase in ionic strength that brings us (at a fixed $\mathrm{pH}$ ) from 2-phase to 1-phase state $\Delta I_{\text {dissol }}$, that is, coacervate dissolution, and then compare this to the increase in protein charge (in the positive direction) that would, at fixed ionic strength, accomplish the same change. We then compare this to the change in protein charge needed to compensate for a loss of binding resulting from the same $\Delta I_{\text {dissol. }}$. We begin by measuring the loss of turbidity with decreasing $\mathrm{pH}$ as shown in Figure 7B. While Figure 7A indicates a critical condition for coacervate dissolution at $\mathrm{pH} 7.15$ of $I^{1 / 2}=0.15 \rightarrow 0.17(22 \rightarrow 29 \mathrm{mM})$, Figure 7B indicates a corresponding condition (with $\tau$ decreasing from 65 to 15$)$ for $\mathrm{pH} 8.0 \rightarrow 6.5$ at fixed $I^{1 / 2}=0.17(29 \mathrm{mM})$. These two conditions overlap fully the same $\mathrm{pH}$ versus $I^{1 / 2}$ phase space. To the extent that equal changes in turbidity correspond to identical changes in state, it is therefore possible to identify a change in $I$ that will exactly compensate for a change in protein charge: increasing $I$ from 22 to $29 \mathrm{mM}\left(\Delta I_{\text {dissol }}\right)$ has an effect on coacervation dissolution equivalent to reducing the protein charge from -20 to -5 (when $\mathrm{pH}$ decreases from 8.0 to 6.5 ). Thus, $\Delta I_{\text {dissol }}$ appears quite small compared to the change in protein charge. We can compare results to the change in protein charge needed to compensate for a reduction in binding arising from the same $\Delta I_{\text {dissol }}$ from values of $\mathrm{d}\left(Z_{\mathrm{pr}}\right)_{\mathrm{c}} / \mathrm{d}(I)$ obtained elsewhere; increasing $I$ from 22 to $29 \mathrm{mM}$ has an effect on binding equivalent to increasing protein positive charge from +40 to $+44 .{ }^{51}$ Thus, compensating for a given increase in ionic strength requires a charge diminution of only 4 charges in the case of binding but requires a charge diminution of 15 charges in the case of coacervate dissolution. Put differently, a given absolute change in protein charge has a significantly larger effect on binding than on coacervation. While the differences in the mechanisms (and surely the kinetics) of coacervation versus binding may preclude simple interpretation, one possible inference is that a change of, for example, $\Delta z_{\mathrm{pr}}=-1$ at the polyelectrolyte binding site has a large effect on $K_{\mathrm{b}}$ compared to its small effect on the net complex charge which governs coacervation.

4. Effect of Stoichiometry. Scheme 1 indicates that a region of complex charge neutrality corresponding to a critical number of bound proteins $n^{*}{ }_{\mathrm{pr}} \approx\left|Z_{\mathrm{p}} / Z_{\mathrm{pr}}\right|$ can be transited by changing the ionic strength $I$ at fixed $\mathrm{pH}$ and bulk stoichiometry $r$. The same scheme suggests that this regime can be traversed by an increase in $r$ at fixed $\mathrm{pH}$ and $I$. Because Figure 2 indicates that $10 \mathrm{mM} \mathrm{NaCl}, \mathrm{pH} \approx 7$, and $r=5$ is a condition of incipient coacervation, we titrated PDADMAC with BSA (increasing $r$ ) at $\mathrm{pH} 7.86 \pm 0.5$ and $I=10 \mathrm{mM}$. As seen in Figure 8 coacervation occurs abruptly at $r^{*}=5$. While $r$ represents the bulk stoichiometry, not necessarily equal to the microscopic stoichiometry, this finding can be correlated with the charge stoichiometry of the complex, as noted in ref 55 in which the addition of cationic protamines to either DNA or poly(styrenesulfonate) resulted in formation followed by dissolution of a dense phase. That this occurred as the (bulk) charge molar ratio progressed from 0.75 to 1.25 strongly suggests that these effects correspond to changes in the complex. To estimate a priori the value of $r$ at which coacervate is destabilized (i.e., $n \gg n^{*}$ pr) we used previous results obtained with a broad distribution (commercial) PDADMAC for $I=10 \mathrm{mM}, \mathrm{pH}=7.9$, and $C_{\mathrm{pr}}$ $=0.06 \mathrm{~g} / \mathrm{L} .{ }^{52}$ Those results suggested $r \approx 10$ as the condition

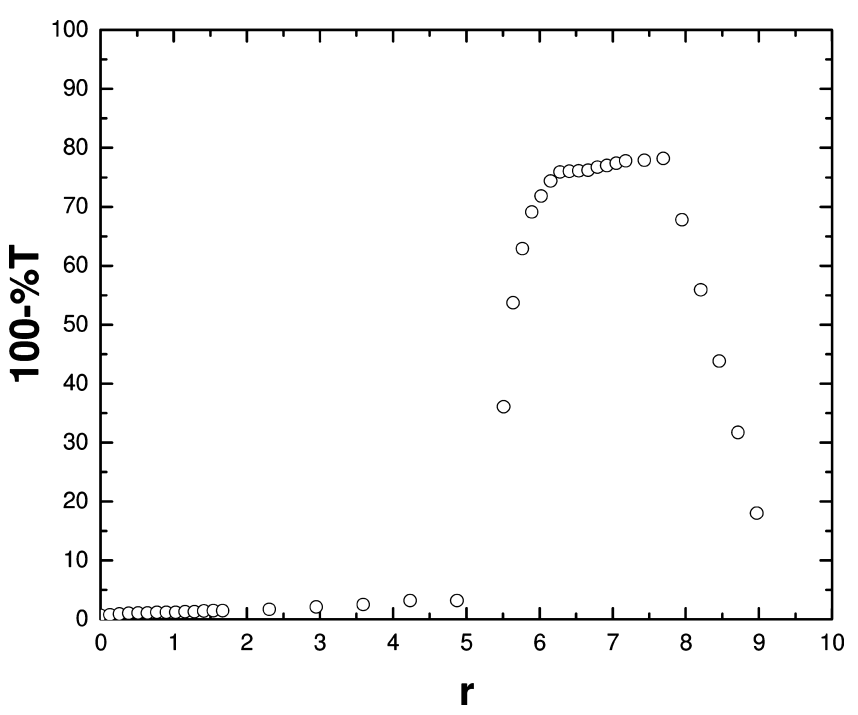

Figure 8. Formation and dissolution of coacervate at $I=10 \mathrm{mM}, \mathrm{pH}$ $=7.9$, upon addition of protein $(20 \mathrm{~g} / \mathrm{L}$ ) to PDADMAC (initial concentration $0.12 \mathrm{~g} / \mathrm{L})$.

of "excess protein", although the transition was broad, attributable in part to the polyelectrolyte polydispersity. The results in Figure 8 are consistent with this expectation. The asymmetry of Figure 8 (lower slope on the high- $r$ side) of the curve may arise from the shape of the binding isotherm seen in Scheme 1, for which $\left(\mathrm{d} n_{\mathrm{pr}} / \mathrm{d} r\right)$ is smaller at high $r$, corresponding to a more gradual change in $Z_{\mathrm{T}}=Z_{\mathrm{P}}-n_{\mathrm{pr}} Z_{\mathrm{pr}}$ with respect to $r$. The fact that stable values of the turbidity are rapidly achieved in the coacervate-dissolution region $r>7.5$ make this explanation more likely than one based on the kinetics of coacervate dissolution. Regardless of this, the transition at " $r$ " is notably less welldefined that the one at $\mathrm{pH}_{\varphi}$, possibly suggesting a strong dependence of protein binding affinity with $\mathrm{pH}$. In support of this, we have observed that a change in BSA ionization of only 5 charges (in the direction opposite to polyelectrolyte charge) requires an increase in $I$ from 10 to $150 \mathrm{mM}$ to nullify the increase in affinity due to protein charge. ${ }^{44}$ This sensitivity to protein charge may suggest that those charges reside in a region that dominates binding energetics.

These results may be compared to the study of the interaction of poly(acrylic acid) with cationic liposomes by Cametti and co-workers. ${ }^{53}$ Well-defined regions of aggregation appeared in the titrations of liposomes with polyelectrolyte at values of $r$ corresponding to charge neutralization. While these aggregate domains appear to be sharp and symmetrical, it should be noted that the plots corresponding to Figure 8 cover 4 orders of magnitude in $r$. At polymer MWs above $2 \times 10,{ }^{5}$ micrometersize particles were seen by TEM high levels of internal structure. Consistent with the results of Shklovskii et al., ${ }^{54}$ such clusters appear to be at equilibrium with smaller complexes.

5. Coacervation Domain at Fixed $\mathbf{p H}$. The limited data available can help to visualize the coacervation domain at fixed $\mathrm{pH}$, that is, as a function of ionic strength $I$ and bulk stoichiometry $r$, as shown for $\mathrm{pH}=7.5 \pm 0.4$ in Scheme 2 . Here we take data from Figure 7A, in which the coacervate region at $r=5$ is entered and then exited at 12 and $25 \mathrm{mM}\left(I^{1 / 2}\right.$ $=0.11$ and 0.16 , respectively) and combine them with results from Figure 8 where entry and exit into the two-phase region are seen for fixed $I$ at $r=0.11$ and 0.16 , respectively. The region enclosed by the curve represents the nonmonotonic behavior of the coacervation doamain. Earlier work done with a polydisperse (commercial) PDADMAC sample confirms such entry 
Scheme 2. Depiction of the Coacervation Domain as Affected by Ionic Strength $(I)$ and Stoichiometric Ratio $(r)^{a}$

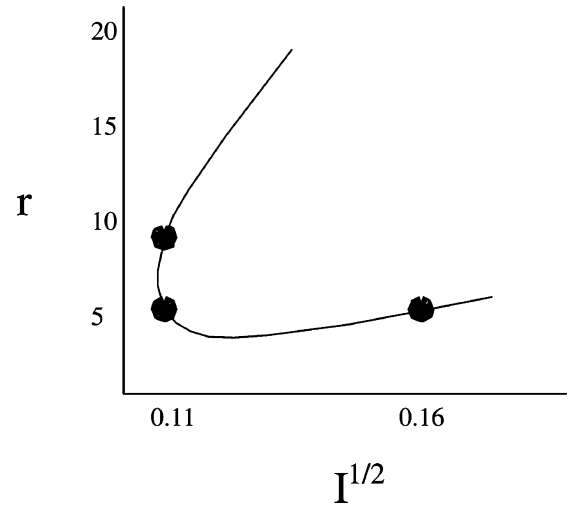

${ }^{a}$ At a fixed $\mathrm{pH}$ of $7.5 \pm 0.4$, using the observed phase changes seen in Figures $7 \mathrm{~A}$ and 8 . The region enclosed by the curve represents the presence of coacervation and illustrates its nonmonotonic behavior.

into and exit from the biphasic region with stoichiometry, ${ }^{52}$ a result consistent with observations from other systems of oppositely charged macroions, for example, DNA with protamines or histones, ${ }^{55}$ Pectin and $\beta$-lactoglobulin, ${ }^{15}$ or $N$-carboxyethyl chitosan and poly(acrylamidomethylpropanesulfonate), ${ }^{56}$ as well as the theoretical treatment of Zhang and Shklovskii, which points to the narrowing of the dense phase domain at low salt. While coacervation may cease to exist at high salt, visualization of a closed loop in Scheme 2 could be an oversimplification, not taking into account the relationship between bulk and microscopic stoichiometry.

Scheme 2 along with Figures 2 and 7A indicate the value of three-dimensional phase boundaries to define the region of coacervate stability with respect to $\mathrm{pH}, I$, and $r$, similar to those constructed for the coacervation of polyelectrolyte-micelle complexes. ${ }^{57,58}$ Of further interest are effects of polyelectrolyte MW and temperature on such boundaries: an increase in the former is expected to expand the coacervation regime, while the prominent temperature-induced enhancement of coacervation for polyelectrolyte-micelle systems ${ }^{59,60}$ does not appear to be shared by polyelectrolyte-protein systems, with no effect of temperature on $\mathrm{pH}_{\varphi}$ for the PDADAMAC-BSA system between 5 and $50{ }^{\circ} \mathrm{C} .{ }^{61}$ It is certainly possible that within these boundaries may lie other transitions, for example, from coacervate to amorphous solid, as suggested by observations for both polyelectrolyte- protein $^{61}$ and polyelectrolyte - micelle $^{59}$ systems.

\section{Conclusions}

We have observed a minimum with respect to ionic strength for the phase boundary for $\mathrm{pH}$-induced coacervation of BSA with poly(dimethyldiallylammonium chloride), and this minimum coincides with the minimum observed for the ionic strength dependence of the critical $\mathrm{pH}$ for complexation. We propose that the correspondence of these two minima may arise from the nonmonotonic effect of ionic strength on binding affinity noted in previous work for a number of protein-polyelectrolyte systems. Entry into and exit from the coacervation regime with changes in ionic strength are demonstrated directly, and we draw attention to how such effects can be related to transit through the coacervation regime induced by changes in protein/polyelectrolyte bulk stoichiometry. This relationship is best understood by recognizing that the formation of coacervate involves the involvement of well-defined species which are likely to be complexes of net charge $Z_{\mathrm{T}}$ close to zero. For polyelectrolytes whose charges are not $\mathrm{pH}$ dependent ("quenched"), $Z_{\mathrm{T}}$ is governed only by protein charge and the number of proteins bound per polymer chain. Because this last quantity depends on $I, r$, and $\mathrm{pH}, Z_{\mathrm{T}}$ can pass through zero as a function of any of these three variables, and the system will undergo transitions into and out of the two phase region. Transitions out of the coacervate domain ("coacervate suppression") display more complex kinetics and less reversibility than coacervate formation. Polyelectrolyte chain stiffness appears to enhance coacervation, but in the present study this effect is not easily separated from the charge mobility of "annealed" polyelectrolytes.

Acknowledgment. We thank Dr. Sabina Strand for providing the chitosan sample.

\section{References and Notes}

(1) Oparin, A. I.; Synge, A. The origin of life on the earth, 3rd ed.; Academic Press: New York, 1957.

(2) Veis, A.; Bodor, E.; Mussell, S. Biopolymers 1967, 5, 37-59.

(3) Overbeek, J. T.; Voorn, M. J. J. Cell. Physiol. Suppl. 1957, 49, 7-22, discussion, 22-26.

(4) Mattison, K. W.; Dubin, P. L.; Brittain, I. J. J. Phys. Chem. B 1998, 102, 3830-3836.

(5) Weinbreck, F.; Nieuwenhuijse, H.; Robijn, G. W.; de Kruif, C. G. Langmuir 2003, 19, 9404-9410.

(6) Xia, J. L.; Dubin, P. L.; Kim, Y.; Muhoberac, B. B.; Klimkowski, V. J. J. Phys. Chem. 1993, 97, 4528-4534.

(7) Singh, S. S.; Siddhanta, A. K.; Meena, R.; Prasad, K.; Bandyopadhyay, S.; Bohidar, H. B. Int. J. Biol. Macromol. 2007, 41, 185-192.

(8) Park, J. M.; Muhoberac, B. B.; Dubin, P. L.; Xia, J. L. Macromolecules 1992, 25, 290-295.

(9) Wen, Y. P.; Dubin, P. L. Macromolecules 1997, 30, 7856-7861.

(10) Zhang, R.; Shklovskii, B. T. Phys. A (Amsterdam, Neth.) 2005, 352, 216-238.

(11) Mattison, K. W.; Brittain, I. J.; Dubin, P. L. Biotechnol. Prog. 1995, 11, 632-637.

(12) Seyrek, E.; Dubin, P. L.; Tribet, C.; Gamble, E. A. Biomacromolecules 2003, 4, 273-282.

(13) Laos, K.; Brownsey, G. J.; Ring, S. G. Carbohydr. Polym. 2007, 67, $116-123$.

(14) Donati, I.; Borgogna, M.; Turello, E.; Cesaro, A.; Paoletti, S. Biomacromolecules 2007, 8, 1471-1479.

(15) Sperber, B. L. H. M.; Schols, H. A.; Stuart, M. A. C.; Norde, W.; Voragen, A. G. J. Food Hydrocolloids 2009, 23, 765-772.

(16) Dubin, P. L.; Gao, J.; Mattison, K. Sep. Purif. Methods 1994, 23, 116.

(17) Yu, J.; Liu, H. Z.; Chen, J. Y. Sep. Sci. Technol. 2002, 37, 217-228.

(18) Wang, Y. F.; Gao, J. Y.; Dubin, P. L. Biotechnol. Prog. 1996, 12, 356-362.

(19) Jintapattanakit, A.; Junyaprasert, V. B.; Mao, S.; Sitterberg, J.; Bakowsky, U.; Kissel, T. Int. J. Pharm. 2007, 342, 240-249.

(20) Dumitriu, S.; Chornet, E. Adv. Drug Delivery Rev. 1998, 31, 223246.

(21) Burgess, D. J.; Kwok, K. K.; Megremis, P. T. J. Pharm. Pharmacol. 1991, 43, 232-236.

(22) Santinho, A. J. P.; Ueta, J. M.; Freitas, O.; Pereira, N. L. J. Microencapsulation 2002, 19, 549-558.

(23) Xia, J. L.; Mattison, K.; Romano, V.; Dubin, P. L.; Muhoberac, B. B. Biopolymers 1997, 41, 359-365.

(24) Dautzenberg, H.; Karibyants, N.; Zaitsev, S. Y. Macromol. Rapid Commun. 1997, 18, 175-182.

(25) Schmitt, C.; Sanchez, C.; Desobry-Banon, S.; Hardy, J. Crit. Rev. Food Sci. Nutr. 1998, 38, 689-753.

(26) de Kruif, C. G.; Weinbreck, F.; de Vries, R. Curr. Opin. Colloid Interface Sci. 2004, 9, 340-349.

(27) Kayitmazer, A. B.; Bohidar, H. B.; Mattison, K. W.; Bose, A.; Sarkar, J.; Hashidzume, A.; Russo, P. S.; Jaeger, W.; Dubin, P. L. Soft Matter 2007, 3, 1064-1076.

(28) Kayitmazer, A. B.; Strand, S. P.; Tribet, C.; Jaeger, W.; Dubin, P. L. Biomacromolecules 2007, 8, 3568-3577.

(29) Kayitmazer, A. B.; Seyrek, E.; Dubin, P. L.; Staggemeier, B. A. J. Phys. Chem. B 2003, 107, 8158-8165.

(30) Bohidar, H.; Dubin, P. L.; Majhi, P. R.; Tribet, C.; Jaeger, W. Biomacromolecules 2005, 6, 1573-1585. 
(31) Cooper, C. L.; Dubin, P. L.; Kayitmazer, A. B.; Turksen, S. Curr Opin. Colloid Interface Sci. 2005, 10, 52-78.

(32) Dautzenberg, H.; Gornitz, E.; Jaeger, W. Macromol. Chem. Phys. 1998 199, 1561-1571.

(33) Schipper, N. G. M.; Varum, K. M.; Stenberg, P.; Ocklind, G.; Lennernas, H.; Artursson, P. Eur. J. Pharm. Sci. 1999, 8, 335-343.

(34) Anthonsen, M. W.; Varum, K. M.; Smidsrod, O. Carbohydr. Polym. 1993, 22, 193-201.

(35) Dubin, P. L.; Rigsbee, D. R.; Gan, L. M.; Fallon, M. A. Macromolecules 1988, 21, 2555-2559.

(36) MattisonK. W. DubinP. L. Abstracts of Papers, 207th National Meeting of the American Chemical Society, San Diego, CA, March 13-17, 1994; American Chemical Society: Washington, DC, 1994; 186 CHED.

(37) Weinbreck, F.; de Vries, R.; Schrooyen, P.; de Kruif, C. G. Biomacromolecules 2003, 4, 293-303.

(38) Groenewold, J.; Kegel, W. K. J. Phys. Chem. B 2001, 105, 11702 11709.

(39) Cardinaux, F.; Stradner, A.; Schurtenberger, P.; Sciortino, F.; Zaccarelli, E. EPL 2007, 77.

(40) Stradner, A.; Sedgwick, H.; Cardinaux, F.; Poon, W. C. K.; Egelhaaf, S. U.; Schurtenberger, P. Nature 2004, 432, 492-495.

(41) Archer, A. J.; Wilding, N. B. Phys. Rev. E 2007, 76.

(42) Schlessinger, B. S. J. Phys. Chem. 1958, 62, 916-920.

(43) Menon, M. K.; Zydney, A. L. Anal. Chem. 1998, 70, 1581-1584.

(44) Cooper, C. L.; Goulding, A.; Kayitmazer, A. B.; Ulrich, S.; Stoll, S.; Turksen, S.; Yusa, S.; Kumar, A.; Dubin, P. L. Biomacromolecules 2006, 7, 1025-1035.

(45) Jintapattanakit, A.; Mao, S.; Kissel, T.; Junyaprasert, V. B. Eur. J. Pharm. Biopharm. 2008, 70, 563-571.
(46) Strand, S. P.; Tommeraas, K.; Varum, K. M.; Ostgaard, K. Biomacromolecules 2001, 2, 1310-1314.

(47) Ou, Z; Muthukumar, M. J. Chem. Phys, 2006, 124, 154902.

(48) Ball, V.; Winterhalter, M.; Schwinte, P.; Lavalle, P.; Voegel, J. C.; Schaaf, P. J. Phys. Chem. B 2002, 106, 2357-2364.

(49) Anthonsen, M. W.; Smidsrod, O. Carbohydr. Polym. 1995, 26, 303305.

(50) Anthonsen, M. W.; Varum, K. M.; Hermansson, A. M.; Smidsrod, O.; Brant, D. A. Carbohydr. Polym. 1994, 25, 13-23.

(51) Mattison, K. Ph.D. thesis Purdue University: West Lafayette, IN, 1999.

(52) Ahmed, L. S.; Xia, J. L.; Dubin, P. L.; Kokufuta, E. J. Macromol. Sci., Pure Appl. Chem. 1994, A31, 17-29.

(53) Bordi, F.; Cametti, C.; Diociaiuti, M.; Gaudino, D.; Gili, T.; Sennato, S. Langmuir 2004, 20, 5214-5222.

(54) Nguyen, T. T.; Shklovskii, B. I. J. Chem. Phys. 2001, 115, 72987308.

(55) Raspaud, E.; Chaperon, I.; Leforestier, A.; Livolant, F. Biophys. J. 1999, 77, 1547-1555.

(56) Mincheva, R.; Manolova, N.; Paneva, D.; Rashkov, I. Eur. Polym. J. 2006, 42, 858-868.

(57) Wang, Y. L.; Kimura, K.; Huang, Q. R.; Dubin, P. L.; Jaeger, W. Macromolecules 1999, 32, 7128-7134.

(58) Wang, Y. L.; Kimura, K.; Dubin, P. L.; Jaeger, W. Macromolecules 2000, 33, 3324-3331.

(59) Kumar, A.; Dubin, P. L.; Hernon, M. J.; Li, Y.; Jaeger, W. J. Phys. Chem. B 2007, 111, 8468-8476.

(60) Dubin, P. L.; Li, Y.; Jaeger, W. Langmuir 2008, 24, 4544-4549.

(61) Kaibara, K.; Okazaki, T.; Bohidar, H. B.; Dubin, P. L. Biomacromolecules 2000, 1, 100-107.

BM900886K 